\title{
Transplacental or Enteral Transfer of Maternal Immunization-Induced Antibody Protects Suckling Rats from Type III Group B Streptococcal Infection ${ }^{1}$
}

\author{
HOWARD S. HEIMAN AND LEONARD E. WEISMAN \\ Departments of Pediatrics, Uniformed Services University of the Health Sciences, Bethesda, Maryland; and \\ Waller Reed Army Medical Center, Washington, DC
}

\begin{abstract}
Deficiency of maternal group B streptococcal (GBS) type-specific IgG increases neonatal susceptibility to GBS infection. We asked if immunization-induced maternal type III GBS opsonic antibody transferred prenatally (via placenta) or postnatally (via breast milk) would affect suckting rat survival after GBS infection. Pregnant immunized dams with type III GBS opsonic antibody (20) through $320 \mathrm{dil}^{-1}$ ) and nonimmunized dams without GBS antibody were matched $(n=16)$. Half of each litter was cross-suckled to a matching dam creating four pup groups with different exposure to maternal type III GBS opsonic antibody: none, postnatal, prenatal, and combined (preand postnatal). After infection with type III GBS, group survival $(n)$ was $41 \%(51), 66 \%(47), 98 \%(43)$, and $98 \%$ (47), respectively. Type III GBS opsonic antibody in surviving pups was directly related to their immunized dam's antibody either postnatally $(R=0.85)$, prenatally $(R=$ 0.84 ), or combined ( $R=0.81$ ). Pups exposed postnatally to high titers ( 80 to 320 dilution $^{-1}$ ) of type III GBS opsonic antibody survived more often than those exposed to low titers $\left(20\right.$ to $\left.40 \mathrm{dil}^{-1}\right)(p<0.03)$. Immunization-induced maternal type III GBS opsonic antibody is transferred preand postnatally and results in improved neonatal survival after GBS infection. Survival of pups exposed to postnatal antibody appears related to the concentration of maternal type III GBS opsonic antibody. Breast milk with high titers of GBS type-specific antibody may modify the course of GBS infection. GBS vaccines and strategies could be tested in this model. (Pediatr Res 26:629-632, 1989)
\end{abstract}

\section{Abbreviations}

CFU, colony forming unit

dil $^{-1}$, dilution ${ }^{-1}$

ELISA, enzyme-linked immunosorbent assay

GBS, group B streptococcus

Maternal antibody deficiency of GBS type-specific IgG increases neonatal susceptibility to type-specific GBS infection (1$5)$. This suggests that transplacental transfer of immunizationinduced maternal type-specific GBS antibody may protect neo-

Received April 19, 1989; accepted August 3, 1989.

Correspondence and reprint requests Leonard E. Weisman, M.D., Department of Pediatrics, Uniformed Services University, Bethesda, MD 20814-4799.

Supported in part by the Department of Clinical Investigation. Walter Reed Army Medical Center, Washington, DC under protocol 6055.

I The opinions and assertations contained herein are those of the authors and do not reflect those of the Department of the Army or the Department of Defense. nates from GBS infection. Previous animal studies, however, do not all demonstrate improved survival associated with transplacental transfer of GBS type-specific antibody (6-8). In addition, these few reported studies did not immunize mothers during pregnancy, determine the impact of immunization on GBS typespecific opsonic antibody, or the effect of prenatally (transplacental) versus postnatally (breast milk) acquired antibody on their neonates. To address these issues we conducted experiments to determine whether intrapartum maternal immunizationinduced type III GBS opsonic antibody transferred prenatally (via placenta) or postnatally (via breast m;lk) would affect suckling rat survival after oral inoculation with type III GBS.

\section{MATERIALS AND METHODS}

Organism. GBS strain S\$878 (virulent, type III clinical isolate) kindly supplied by Dr. Gerald Fischer of the Uniformed Services University of the Health Sciences, Bethesda, MD was grown to midlogarithmic phase in Todd Hewitt broth and stored at $-70^{\circ} \mathrm{C}$ until used.

Immunization preparation. The organism was cultured overnight on Columbia sheep blood agar (BBL Microbiologic Systems, San Juan Capistrano, CA), incubated 12 to $18 \mathrm{~h}$ in $25 \mathrm{~mL}$ of Todd Hewitt broth, washed twice with normal saline, and resuspended in $0.2 \%$ formalin. The suspension was stored at $4{ }^{\circ} \mathrm{C}$ until sterility was confirmed. The formalin-killed bacteria were washed and resuspended in normal saline to a concentration of $2 \times 10^{9} \mathrm{CFU} / \mathrm{mL}$ and stored at $-70^{\circ} \mathrm{C}$ (9)

Animal studies. Eighteen nulliparous, barrier reared, Wistar rat dams whose sera were negative for type III GBS antibody using an ELISA (10) were provided by a commercial breeder (Charles River Breeding Laboratories, Wilmington, MA). Vaginal plug positive dams mated during a common 24-h period were randomly assigned (using a table of random numbers) to the immunized or nonimmunized group (Table 1). Animals were housed in separate stainless steel cages with hardwood litter, under uniflow filtered hoods and fed antibiotic-free Purina Rat Chow (Ralston Purina, Co., St. Louis, MO) and water ad libitum.

Immunized dams were given $1 \mathrm{~mL}$ of formalin-killed type III GBS $\left(1 \times 10^{9} \mathrm{CFU}\right)$ emulsified $1: 1$ in Freund's adjuvant. Complete adjuvant was used for the primary injection in the footpads at $3 \mathrm{~d}$ postconception, and incomplete adjuvant was used for intramuscular boosters at 11 and $18 \mathrm{~d}$ postconception (11).

Litters from an immunized and nonimmunized mother were matched to minimize the difference between the two litters' birth times. All matched litters delivered less than $6 \mathrm{~h}$ apart and contained seven to 14 pups. The pups in each litter were randomly divided (using a table of random numbers) into two groups. One group suckled on their own mother, whereas the other group suckled on the opposite matched mother (Table 1). 
Table 1. Experimental design

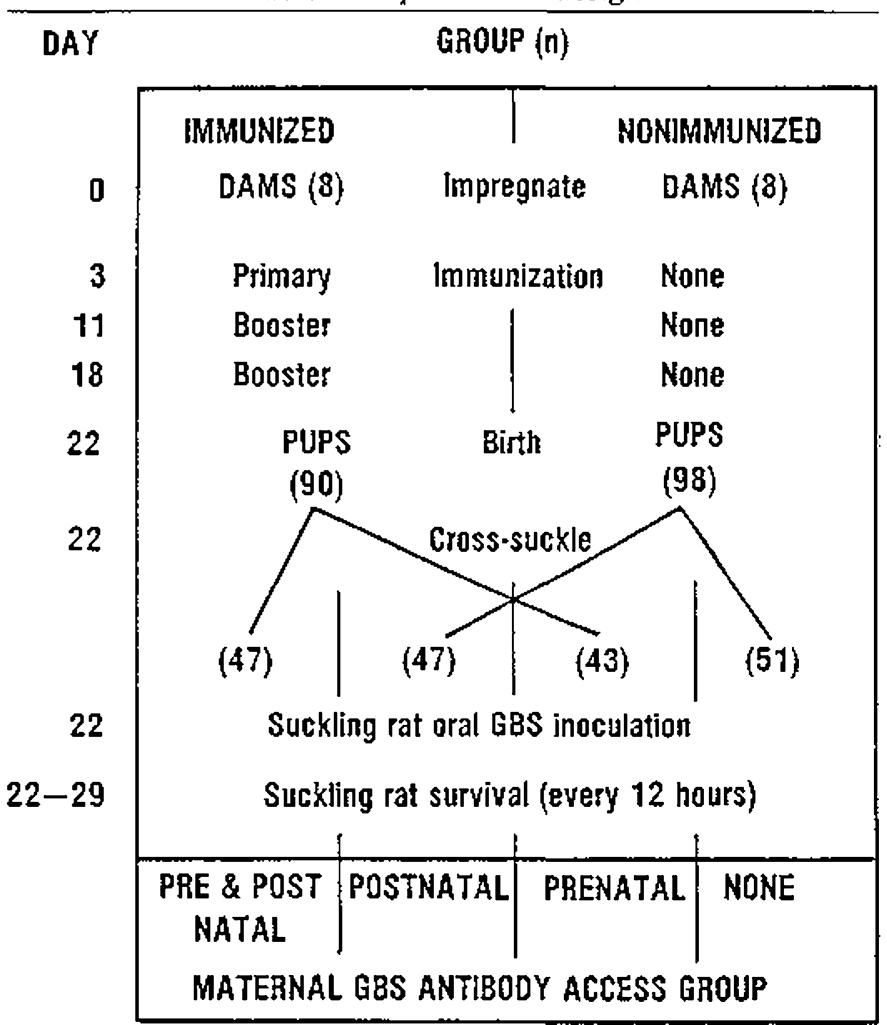

The four experimental groups created had either. 1) prenatal, 2) postnatal, 3) combined (pre- and postnatal), or 4) no maternal immunization-induced type III GBS antibody exposure. All pups were orally inoculated twice with $10^{8} \mathrm{CFU} / \mathrm{mL}$ of live type III GBS ( $2 \mathrm{~h}$ apart)(12). Pup survival was determined every $12 \mathrm{~h}$ for $8 \mathrm{~d}$.

Serologic assays. Serum was collected from each dam by tail bleed before immunization and at delivery. On the 8th d, pup serum was collected by cardiac puncture using ether anesthesia and pooled by litter and group (prenatal, postnatal, combined, or none). All sera were stored at $-70^{\circ} \mathrm{C}$ and then analyzed for: I) total rat IgG by radial immunodiffusion (done in duplicate, with sheep anti-rat IgG, ICN Immunobiologicals, Lisle, IL) (13), and 2) type III GBS opsonophagocytic antibody (14). The latter assay measures antibody mediated killing of type III GBS in the presence of human polymorphonuclear leukocytes and GBS antibody free infant rabbit complement in vitro. The results are expressed as the highest serum $\mathrm{dil}^{-1}$ that causes killing of $\geq 90 \%$ of GBS (type III, strain SS878). Before testing for opsonic antibody, all rat sera were heat inactivated at $56^{\circ} \mathrm{C}$ for $30 \mathrm{~min}$. Controls for each assay included neutrophils alone, complement alone, neutrophils plus complement, and a $5 \%$ preparation of human intravenous immunoglobulin (Sandoglobulin, Sandoz Inc, East Hanover, $\mathrm{NJ}$ ) with previously determined levels of opsonic antibody.

Statistical analysis. The critical ratio of proportions was used to calculate the minimum number of pups required in each study group. Assuming an orally inoculated pup survival rate of $48 \%$ (12), the estimated sample size required to detect an increase in survival to $70 \%$ with an $\alpha$ error of $5 \%$ and a $\beta$ error of $20 \%$ was 39. The experiments were continued until all study groups exceeded this size.

Fisher's exact test was used to compare the pup survival between the four study groups. Linear regression and Pearson's correlation coefficient were used to analyze the relationship of pup opsonic antibody or maternal total IgG to maternal opsonic antibody levels at birth or maternal total IgG on pup survival. Friedman's method was used to conduct a two-way layout analy- sis of the effect of maternal opsonic antibody concentration on pup survival (15). Wilcoxon's rank sum test was used for litter size comparisons. The Student's two-tailed $t$ test was used in comparisons of paired and independent means. Where appropriate, results are expressed as mean $\pm \mathrm{SD}$. The institutional Animal Use Committee approved the experimental protocol.

\section{RESULTS}

Eighteen dams (nine nonimmunized and nine immunized) were entered into the study and 16 matched. One nonimmunized dam was not pregnant so one immunized dam was excluded. No dams had type III antibody detectable by ELISA (10) or opsonophagocytosis (14) before immunization. Neither did nonimmunized dams at delivery. Immunized dams developed opsonic antibody $\geq 20 \mathrm{dil}^{-1}$ (range from 20 to $320 \mathrm{dil}^{-1}$ ). Total IgG on the day of birth for the immunized dams $(439 \pm 257 \mathrm{mg} / \mathrm{dL})$ was not significantly different than the nonimmunized dams $(273 \pm 115 \mathrm{mg} / \mathrm{dL})(p>0.10)$. There was no correlation of the immunized dams' total IgG with their titer of type II GBS opsonic antibody $\left(R^{2}=0.06, p=0.55\right)$ or with their pups' survival ( $\left.R^{2}=0.003, p=0.64\right)$.

The time from birth to cross-suckling was $3.2 \pm 1.9 \mathrm{~h}$ (range $1.0-7.8)$ and from cross-suckling to inoculation was $6.1 \pm 2.0 \mathrm{~h}$ (range 3.9-10.1) without significant differences between the four groups of pups. The litter size of immunized dams $(11.3 \pm 2.8$, range $7-14$ ) was not significantly different than nonimmunized dams $(12.3 \pm 1.4$, range $10-14)$. There was no evidence of maternal rejection of the cross-suckled pups.

The effect of antibody exposure on pup survival is illustrated in Figure 1. Pups without exposure to maternal antibody had a

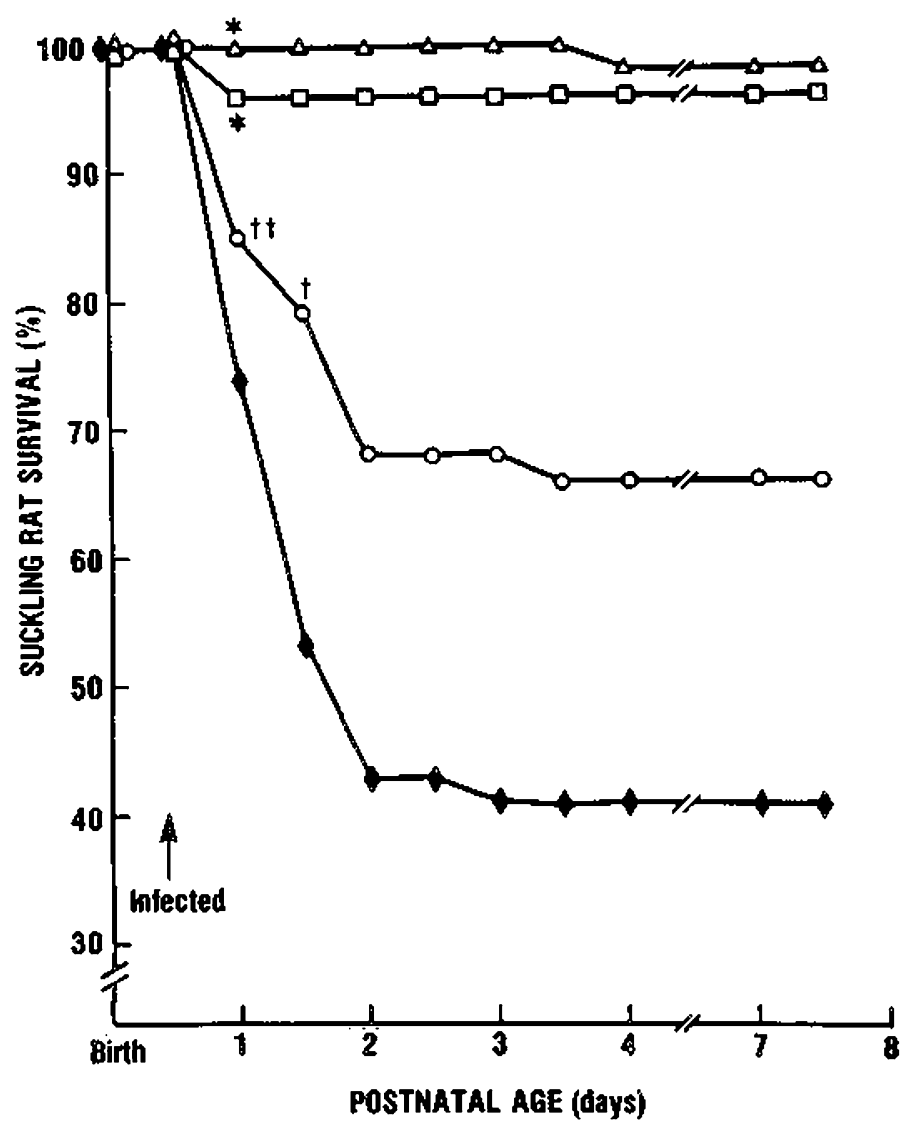

Fig. 1. Survival of GBS-infected suckling rats exposed to prenatal (square, $n=43$ ), postnatal (circle, $n=47$ ), combined (pre- and postnatal, iriangle, $n=47$ ), or to no immunization-induced type III GBS opsonic antibody (diamond, $n=51$ ). ${ }^{*} p \leq 0.001$ versus no antibody, ${ }^{+4} p<0.05$ verstus prenatal or combined antibody, ${ }^{+} p<0.01$ versus no antibody. 